\title{
Association of $\mathrm{FOSB}$ exon 4 unmethylation with poor prognosis in patients with late-stage non-small cell lung cancer
}

\author{
DONG SUN KIM ${ }^{1}$, WON KEE LEE ${ }^{2}$ and JAE YONG PARK ${ }^{3}$ \\ Departments of ${ }^{1}$ Anatomy and BK21 Plus KNU Biomedical Convergence Program, \\ ${ }^{2}$ Preventive Medicine, and ${ }^{3}$ Internal Medicine, School of Medicine, \\ Kyungpook National University, Daegu 702-422, Republic of Korea
}

Received April 18, 2019; Accepted November 25, 2019

DOI: 10.3892/or.2019.7431

\begin{abstract}
Alterations in DNA methylation have a central role in the development and outcome of most human malignancies. Non-small cell lung cancer (NSCLC), the most common lung cancer, leads to the largest number of cancer-related deaths worldwide. FBJ murine osteosarcoma viral oncogene homolog B (FOSB) is a key component of the activator protein-1 transcription factor and regulates gene networks associated within oncogenic transformation. The role of FOSB in the development of NSCLC is still elusive. Therefore, the methylation status of the FOSB gene was investigated in NSCLC and its clinical significance in NSCLC progression was evaluated. The methylation status of the promoter and exon 4 regions of the FOSB gene were analyzed in 176 NSCLC specimens by bisulfite pyrosequencing and the association between FOSB methylation status and patient survival was investigated. Compared to adjacent non-malignant tissues, FOSB promoter exhibited exclusive unmethylation in all malignant tissues and the exon 4 region was found unmethylated in 18 (10.2\% of the total) tumor samples. Exon 4 unmethylation was associated with downregulation of its mRNA and tended to occur in patients with lymph node metastasis. Univariate and multivariate analyses revealed that exon 4 unmethylation was significantly associated with unfavorable overall survival in patients with stage II-IIIA NSCLC (log-rank $\mathrm{P}=0.05$, adjusted hazard ratio $=2.43,95 \%$ confidence interval=1.04-5.68, $\mathrm{P}=0.04) . F O S B$ was identified as a novel gene with tumor-specific gene body unmethylation in NSCLC and a novel predictive biomarker for NSCLC prognosis. Moreover, the present results indicated that FOSB
\end{abstract}

Correspondence to: Dr Jae Yong Park, Department of Internal Medicine, School of Medicine, Kyungpook National University, 2-101 Dongin-dong, Jung-gu, Daegu 702-422, Republic of Korea E-mail: jaeyong@knu.ac.kr

Key words: promoter, exon, unmethylation, FOSB, NSCLC, prognosis may have a tumor suppressor function in the progression of NSCLC.

\section{Introduction}

Non-small cell lung cancer (NSCLC) represents approximately $85 \%$ of all lung cancer cases and remains the leading cancer killer with globally increasing incidence (1). Despite public and medical endeavors to vanquish this disease, NSCLC has risen during the last decade, and $>40 \%$ of the patients diagnosed with NSCLC have distant metastases, which are associated with unsatisfactory prognosis and high mortality (2). NSCLC is also considered as a highly heterogeneous disease that differs in epidemiological, histological, molecular and phenotypic characteristics (3), rendering it an urgent challenge for early detection and effective treatment of disease. Nowadays, it has become clear that epigenetic and genetic modifications occurring in a number of genes play an important role for the maintenance of normal cellular homeostasis and in the occurrence of numerous pathologies; on the other hand, the intervention on such modifications may improve the treatment and prevention of numerous diseases, including NSCLC.

DNA methylation is a fundamental process for eukaryotic development and leads to long term-repression of genome expression (4). DNA methylation changes are common and relatively stable in various types of cancers, providing useful tools for diagnostic, prognostic and even therapeutic intervention (5). It has been reported that a wide spectrum of aberrantly methylated genes in NSCLC regulate proliferation and maintenance of genome stability $(6,7)$. Notably, tobacco smoking, a major etiological factor for NSCLC, may predispose to aberrant methylation of key regulatory genes (8).

FBJ murine osteosarcoma viral oncogene homolog B (FOSB), a member of the FOS family, heterodimerizes with JUN family proteins to form activator protein-1 (AP-1) transcription factor complexes, which play a central role in the transcriptional regulation of numerous genes that are associated with cell proliferation, differentiation, migration, metastasis, and apoptosis (9). Recent data indicated that FOSB 
can act as a common target for anticancer drugs $(10,11)$. In contrast to the amount of data on the function of c-FOS, far less is known about the role of FOSB (12). In addition, despite the numerous studies that have revealed its oncogenic function in tumor formation (13-17), FOSB has been revealed to be downregulated in breast, gastric, and pancreatic cancer $(18,19,20)$. Furthermore, there are controversial data reporting the expression and functional role of FOSB in lung cancer $(11,21)$. Therefore, to understand the biological role of FOSB and its clinical significance in NSCLC progression, the methylation status of the promoter and exon 4 of the FOSB gene was evaluated in 176 specimens from patients with NSCLC using bisulfite sequencing and its association with patient outcomes was investigated.

\section{Materials and methods}

NSCLC tissue samples. Fresh tissue samples were obtained from 176 patients (aged 35 to 83 years) with primary NSCLC and corresponding normal tissues. The present study was approved by the Institutional Review Board (IRB) of Kyungpook National University Hospital (KNUH; Daegu, Korea) (No. 2014-04-210) and all patients provided signed informed consent. The clinical characteristics of the patients are summarized in Table I. NSCLC and normal tissue samples were obtained at the time of surgery, and were immediately frozen in liquid nitrogen and stored at $-80^{\circ} \mathrm{C}$ until DNA extraction. Only tumors with $>80 \%$ of tumorous components were used for methylation analysis and the histological adequacy of tissue specimens was verified by hematoxylin-eosin (H\&E) staining. Tumors were staged according to the American Joint Cancer Committee (AJCC) criteria.

Cell culture, total RNA isolation, and reverse transcription PCR (RT-PCR). The NCI-H157 and NCI-H187 cell lines were obtained from the American Type Culture Collection and cultured in Dulbecco's modified Eagle's medium/F12 or RPMI-1640 medium (Thermo Fisher Scientific, Inc.) with $10 \%$ fetal bovine serum and $1 \%$ penicillin-streptomycin. Genetic characteristics of the NCI-H157 cell line were determined by PCR-single-locus-technology at Eurofins Genomics. Cells were maintained in an incubator at $37^{\circ} \mathrm{C}$ in $5 \% \mathrm{CO}_{2}$. 5-Aza-2'-deoxycytidine (5-AzadC) was added daily to the culturing medium at the indicated concentration for $72 \mathrm{~h}$. Total RNA was isolated from clinical tissue samples and cell lines using TRIzol (Thermo Fisher Scientific, Inc.) and then first-strand cDNA was synthesized using the SuperScript First-Strand Synthesis Kit (Thermo Fisher Scientific, Inc.) according to the manufacturer's protocol. The relative expression of FOSB mRNA was measured with semi-quantitative RT-PCR using GAPDH as an internal control for normalization. All primer sequences are listed in Table SI. The PCR thermal cycling began with initial denaturation for $2 \mathrm{~min}$ at $95^{\circ} \mathrm{C}$, followed by 30 amplification cycles $\left(1 \mathrm{~min}\right.$ at $95^{\circ} \mathrm{C}$, $1 \mathrm{~min}$ at $54^{\circ} \mathrm{C}, 1 \mathrm{~min}$ at $72^{\circ} \mathrm{C}$ ) and a final $5 \mathrm{~min}$ incubation at $72^{\circ} \mathrm{C}$. PCR products were electrophoresed on $2 \%$ agarose gel, stained with ethidium bromide $(0.5 \mu \mathrm{g} / \mathrm{ml})$ for $15 \mathrm{~min}$ at room temperature and visualized using the Syngene DigiGenius Gel Documentation system (Syngene).
DNA extraction, bisulfite treatment, and methylation analysis. DNA samples were extracted using the QIAamp DNA Mini Kit (Qiagen, Inc.) and bisulfited using the EZ DNA Methylation-Gold Kit (Zymo Research) following the manufacturer's instructions. The converted DNA was pyrosequenced using a PyroMark Q96MD system (Qiagen, Inc.) as previously described (22). PCR primer sequences and pyrosequencing primers are presented in Table SI. FOSB methylation for each sample was calculated from the average value of examined CpGs [mC/total C x100 (\%)] and represented as a mean methylation index (MI).

Statistical analysis. Differences in the methylation level of FOSB promoter and exon 4 between tumor and matched normal tissues were analyzed using paired t-tests. A comparison of unmethylation proportion of FOSB exon 4 according to the clinical characteristics was evaluated using chi-square test. Overall survival (OS) was estimated with Kaplan-Meier method (log-rank test) and multivariate Cox regression analysis. A P-value $<0.05$ was considered to indicate a statistically significant difference. All analyses were performed using SAS ver. 9.4 (SAS, Inc.).

\section{Results}

Methylation status and expression of the FOSB gene in NSCLC samples. Bisulfite pyrosequencing was performed to analyze the methylation status of the human FOSB gene in malignant and adjacent non-malignant lung tissue of 176 NSCLC patients. Due to DNA methylation changes in the promoter and gene body regions of $F O S B(23,24)$, pyrosequencing primers encompassing nine and seven $\mathrm{CpGs}$ within the promoter and exon 4 regions of the FOSB gene were designed, respectively. Representative examples of pyrosequencing are presented in Fig. 1 and revealed that non-CpG cytosine residues were converted to thymine, indicating the completeness of bisulfite treatment. The present results revealed that there were no statistically significant differences of the mean DNA methylation level of FOSB promoter $(\mathrm{P}=0.089)$ and exon $4(\mathrm{P}=0.175)$ between tumor and matched normal tissues (Fig. 2). The CGI of the FOSB promoter region was completely unmethylated in all nonmalignant and malignant lung tissues. However, compared to the MI of normal tissues, the DNA methylation level of the FOSB exon 4 region was markedly ( $>2$-folds) decreased in 18 (10.2\% of the total) tumor tissues, indicating that the unmethylation of FOSB exon 4 may be a tumor-associated event during NSCLC tumorigenesis.

It was then investigated whether the unmethylation of FOSB exon 4 could regulate its mRNA expression in representative lung tissue specimens. Methylation status combined with RT-PCR results revealed low or undetectable levels of $F O S B$ transcripts in tumor tissues with unmethylated alleles (213T, 236T and 291T), and high FOSB levels in tumor and non-tumor lung tissues with methylated alleles (Fig. 3A and B). This observation was further substantiated by 5-AzadC treatment of NSCLC cell lines. FOSB mRNA was absent in H187 cells with unmethylated exon 4, and present in H157 cells with methylated alleles; notably, FOSB expression decreased upon 5-AzadC treatment (Fig. 3C). Collectively, these results 
A 205T-promoter

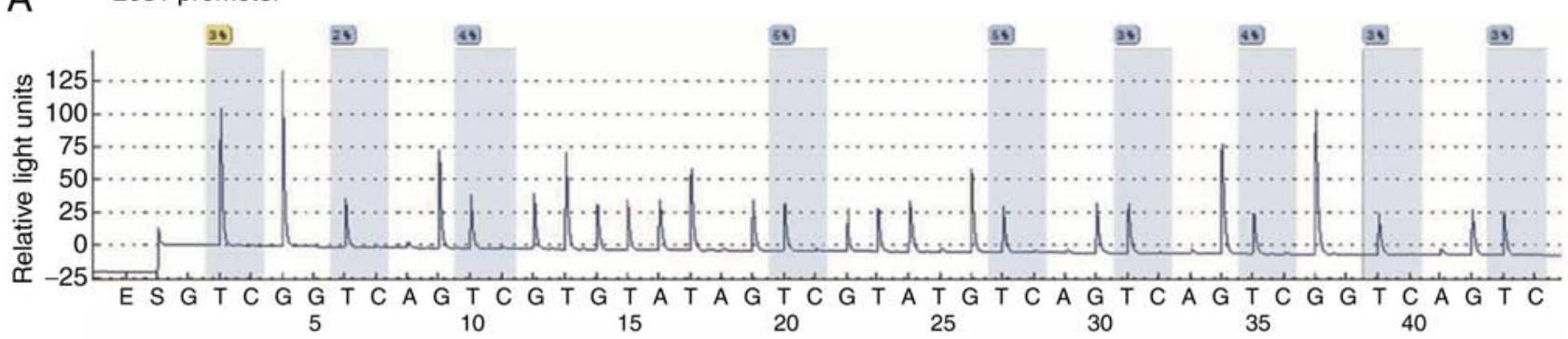

B 213T-promoter

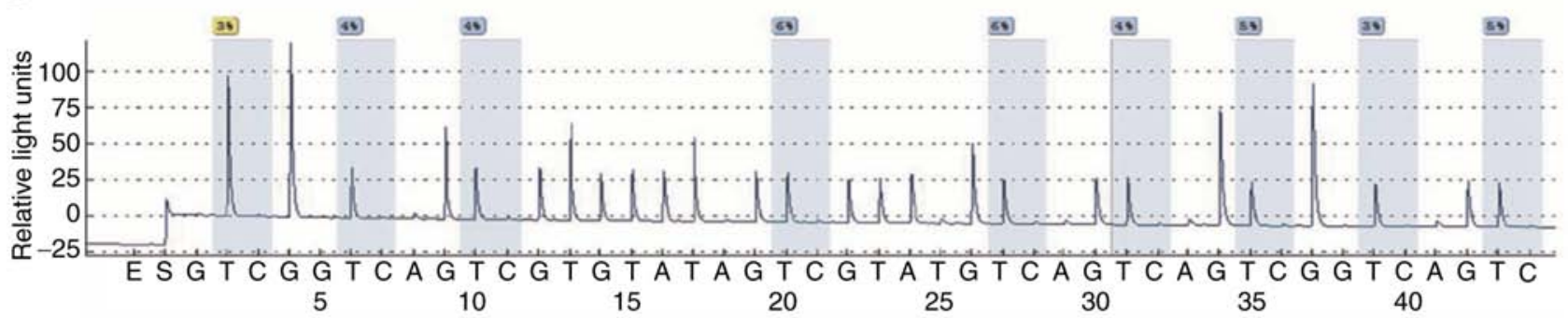

C 205T-exon 4

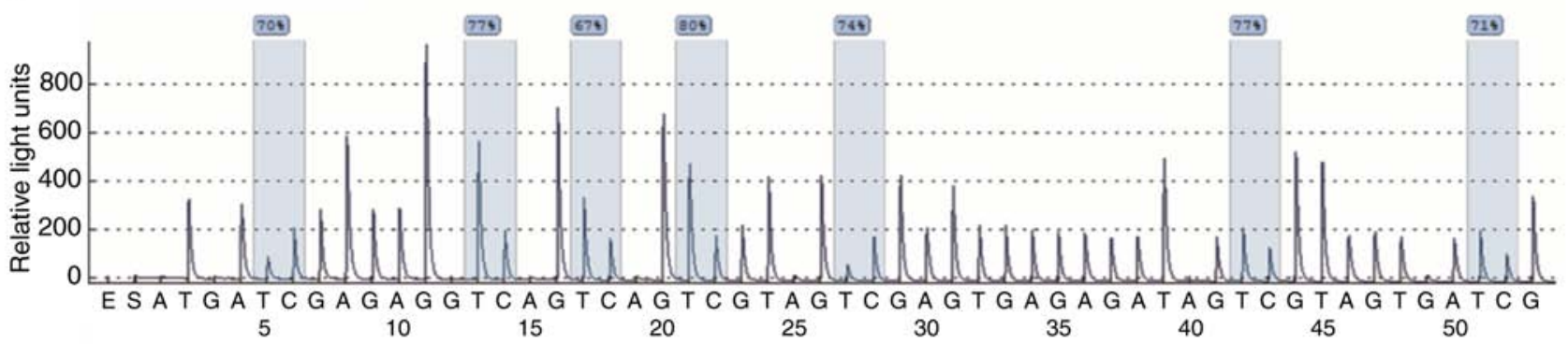

D 213T-exon 4

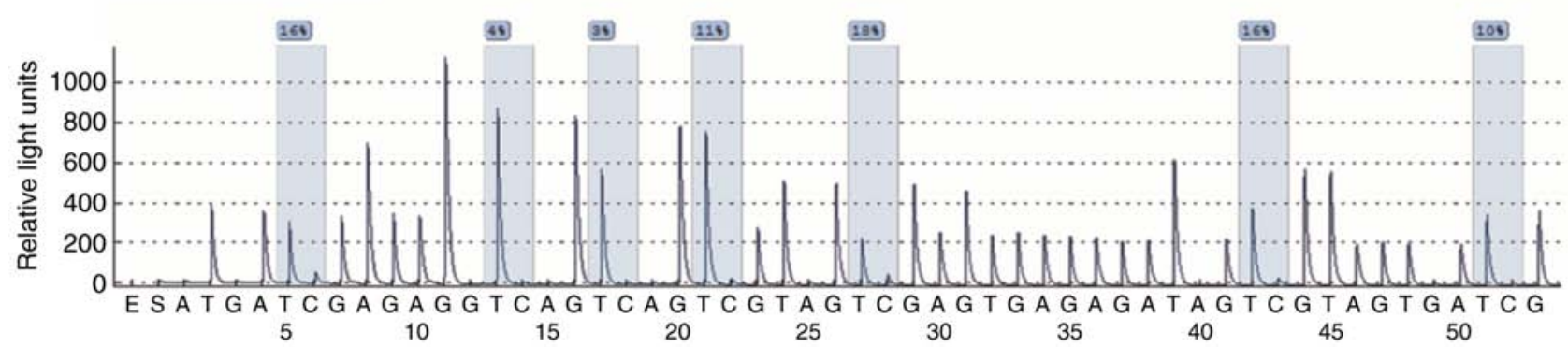

Figure 1. Representative pyrograms of FOSB in lung tissues of NSCLC patients. The methylation status of the (A and B) promoter and (C and D) exon 4 regions of the FOSB gene was determined in NSCLC primary lung tissues using pyrosequencing. The letters on the axis represent the dispensation order; E, enzyme mix; S, substrate; A, G, C, and T, nucleotides. Shaded bars encompassing T/C pairs indicate the eight investigated CpGs. The methylation of each CpG site was calculated as a percentage of C incorporation. FOSB, FBJ murine osteosarcoma viral oncogene homolog B; NSCLC, non-small cell lung cancer.

indicated that exon 4 hypomethylation may be indicative of FOSB gene silencing.

Association of FOSB methylation status with clinicopathological parameters and survival outcome. Unmethylated $F O S B$ exon 4 was more frequent in patients with lymph node metastasis than in those without metastasis with a borderline significance (17.4 vs. $7.7 \%, \mathrm{P}=0.062$; Table I). However, no significant association was revealed in other clinicopathological factors, such as age, sex, smoking status, histology, and pathologic stage (Table I). The methylation status of the FOSB exon 4 in adenocarcinoma and squamous cell carcinoma, respectively, was specifically analyzed (Table SII).

Next, Kaplan-Meier survival analysis was performed to determine the prognostic potential of FOSB methylation status. The patients with unmethylated FOSB had significantly shorter OS than those with FOSB methylation $\left(\mathrm{P}_{\mathrm{L}-\mathrm{R}}=0.045\right.$, Fig. $\left.4 \mathrm{~A}\right)$. After stratification, according to the clinicopathological features, FOSB unmethylation was markedly associated with an unfavorable OS in patients with stage II-IIIA $\left(\mathrm{P}_{\mathrm{L}-\mathrm{R}}=0.056\right.$; Fig. 4B). To evaluate whether FOSB unmethylation is an independent prog- 
Table I. Comparison of methylation status of the FOSB exon 4 according to characteristics of NSCLC patients.

\begin{tabular}{|c|c|c|c|}
\hline Variables & Unmethylation, n (\%) & Methylation, n (\%) & P-value \\
\hline All subjects & $18(10.2)$ & $158(89.8)$ & \\
\hline \multicolumn{4}{|l|}{ Age (years) } \\
\hline$\leq 64$ & $7 \quad(8.9)$ & $72(91.1)$ & 0.589 \\
\hline$>64$ & $11(11.3)$ & $86(88.7)$ & \\
\hline \multicolumn{4}{|l|}{ Sex } \\
\hline Men & $15(12.2)$ & $108(87.8)$ & 0.189 \\
\hline Women & $3(5.7)$ & $50(94.3)$ & \\
\hline \multicolumn{4}{|l|}{ Smoking status } \\
\hline Ever & $15(12.6)$ & $104(87.4)$ & 0.132 \\
\hline Never & $3(5.3)$ & $54(94.7)$ & \\
\hline \multicolumn{4}{|c|}{ Histological types } \\
\hline SQC & $11(13.1)$ & $73(86.9)$ & 0.230 \\
\hline $\mathrm{ADC}$ & $7 \quad(7.6)$ & $85(92.4)$ & \\
\hline \multicolumn{4}{|c|}{ Lymph node metastasis } \\
\hline No & $10(7.7)$ & $120(92.3)$ & 0.062 \\
\hline $\mathrm{N} 1$ and $\mathrm{N} 2$ & $8(17.4)$ & $38(82.6)$ & \\
\hline \multicolumn{4}{|c|}{ Pathologic stage } \\
\hline Stage I & $6(6.6)$ & $85(93.4)$ & 0.100 \\
\hline Stage II-IIIA & $12(14.1)$ & $73(85.9)$ & \\
\hline
\end{tabular}

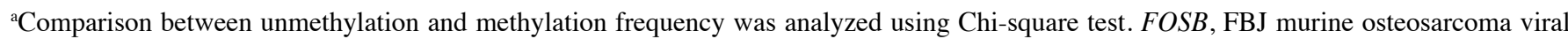
oncogene homolog B; NSCLC, non-small cell lung cancer; SQC, squamous cell carcinoma; ADC, adenocarcinoma.

A

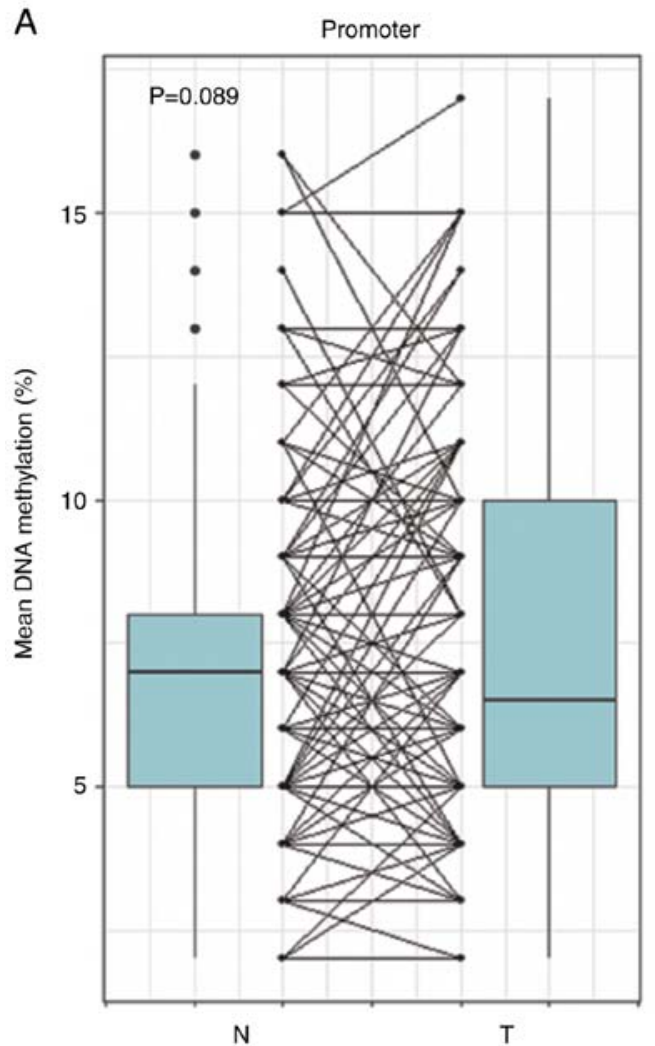

B

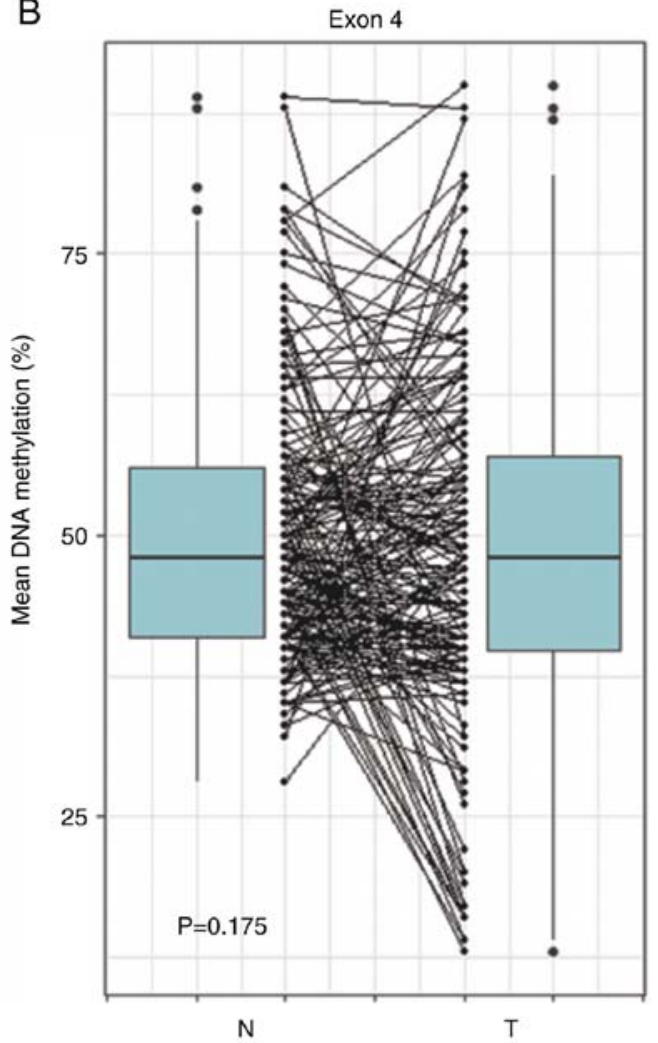

Figure 2. Comparison of the mean methylation level of FOSB between malignant and non-malignant tissues. The mean DNA methylation levels of the tested CpG sites in the FOSB (A) promoter and (B) exon 4 regions were used to compare their differences between NSCLC tumors and the adjacent normal tissues. P-values were obtained using paired t-tests. FOSB, FBJ murine osteosarcoma viral oncogene homolog B; N, adjacent normal tissue; T, tumor tissue; NSCLC, non-small cell lung cancer. 
A Pyrosequencing analysis

\begin{tabular}{|c|c|c|c|c|c|c|c|c|c|c|c|c|}
\hline Tissues & $205 \mathrm{~N}$ & $205 \mathrm{~T}$ & $213 \mathrm{~N}$ & $213 \mathrm{~T}$ & $236 \mathrm{~N}$ & $236 \mathrm{~T}$ & $269 \mathrm{~N}$ & $269 \mathrm{~T}$ & $291 \mathrm{~N}$ & $291 \mathrm{~T}$ & $304 \mathrm{~N}$ & $304 \mathrm{~T}$ \\
\hline Promoter-MI (\%) & 6.7 & 3.7 & 8.1 & 4.4 & 11.2 & 10.0 & 6.6 & 8.9 & 14.6 & 15.4 & 9.3 & 5.9 \\
\hline Exon 4-MI (\%) & 62.9 & 73.7 & 50.9 & 11.1 & 69.4 & 17.4 & 71.5 & 56.7 & 68.5 & 27.6 & 51.4 & 64.9 \\
\hline
\end{tabular}

B RT-PCR analysis

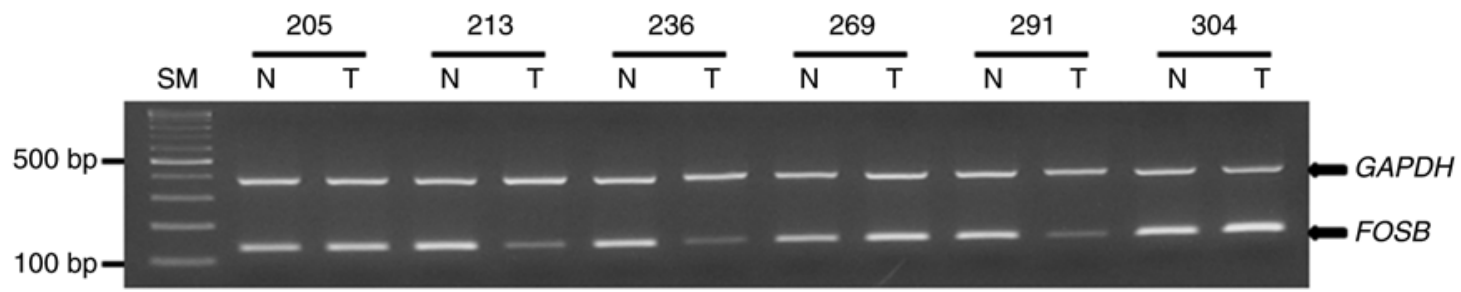

C 5-AzadC treatment

\begin{tabular}{|c|c|c|c|c|}
\hline Cell lines & $\mathrm{H} 187$ & $\mathrm{H} 187$ & $\mathrm{H} 157$ & $\mathrm{H} 157$ \\
\hline 5-AzadC $(\mu \mathrm{M})$ & 0 & 20 & 0 & 20 \\
\hline Promoter-MI (\%) & 8.1 & 8.8 & 12.4 & 9.0 \\
\hline Exon 4-MI (\%) & 21.4 & 17.0 & 86.4 & 28.1 \\
\hline
\end{tabular}

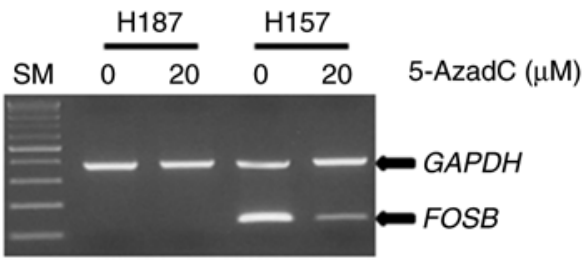

Figure 3. Pyrosequencing and RT-PCR analysis of the FOSB gene in patients with NSCLC and cell lines. FOSB methylation status and mRNA expression were determined by (A) pyrosequencing and (B) semi-quantitative RT-PCR in NSCLC specimens and (C) cell lines. H157 and H187 cell lines were incubated with $20 \mu \mathrm{M}$ 5-AzadC for 3 days. GAPDH was loaded as an internal control for normalization. SM, 100-bp DNA ladder. FOSB, FBJ murine osteosarcoma viral oncogene homolog B; NSCLC, non-small cell lung cancer; MI, mean methylation index; N, adjacent normal tissue; T, tumor tissue; 5-AzadC, 5-aza-2'-deoxycytidine.

A Total patients



B Stage II-IIIA patients

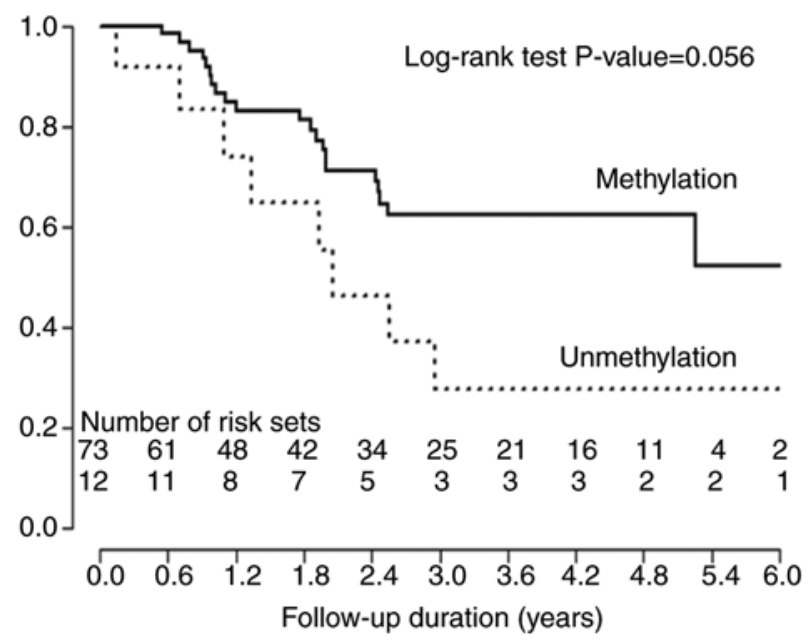

Figure 4. Association of FOSB exon 4 unmethylation with worse survival outcome of NSCLC patients. Kaplan-Meier survival curves for (A) all patients and (B) patients with stage II-IIIA NSCLC according to FOSB methylation status. P-values are based on the log-rank test. FOSB, FBJ murine osteosarcoma viral oncogene homolog B; NSCLC, non-small cell lung cancer.

nostic predictor in NSCLC, the data was further analyzed using the Cox proportional hazards regression model after adjusting for possible cofounding variables of survival. Multivariate analysis revealed that $F O S B$ unmethyl- ation was associated with poor survival in patients with stage II-IIIA [adjusted hazard ratio $(\mathrm{HR})=2.43,95 \%$ confidence interval $(\mathrm{CI})=1.04-5.68, \mathrm{P}=0.040]$, but not in all subjects (Table II). 
Table II. Overall survival according to methylation status of FOSB genes in NSCLC patients.

\begin{tabular}{|c|c|c|c|c|c|c|}
\hline Variables & $\begin{array}{l}\text { No. of } \\
\text { cases }\end{array}$ & $\begin{array}{c}\text { No. of } \\
\text { deaths }(\%)^{\mathrm{a}}\end{array}$ & $\begin{array}{c}\text { 5-year } \\
\text { survival rate }(\%)^{\mathrm{b}}\end{array}$ & $\mathrm{P}_{\mathrm{LR}}^{\mathrm{c}}$ & $\begin{array}{c}\text { Adjusted } \\
\text { HR }(95 \% \text { CI })\end{array}$ & P-value \\
\hline All subjects & 176 & $47(26.7)$ & 61.2 & \multirow{3}{*}{0.045} & & \multirow{3}{*}{0.250} \\
\hline Methylation & 158 & $37(23.4)$ & 64.2 & & 1 & \\
\hline Unmethylation & 18 & $10(55.6)$ & 41.7 & & $1.53(0.74-3.16)^{\mathrm{d}}$ & \\
\hline \multicolumn{7}{|l|}{ Stage I } \\
\hline Methylation & 85 & $16(18.8)$ & 65.8 & \multirow[t]{2}{*}{0.812} & 1 & \multirow[t]{2}{*}{0.905} \\
\hline Unmethylation & 6 & $2(33.3)$ & 66.7 & & $0.91(0.20-4.12)^{\mathrm{e}}$ & \\
\hline \multicolumn{7}{|l|}{ Stage II-IIIA } \\
\hline Methylation & 73 & $21(28.8)$ & 62.5 & \multirow[t]{2}{*}{0.056} & 1 & \multirow[t]{2}{*}{0.040} \\
\hline Unmethylation & 12 & $8(66.7)$ & 27.8 & & $2.43(1.04-5.68)^{\mathrm{e}}$ & \\
\hline
\end{tabular}

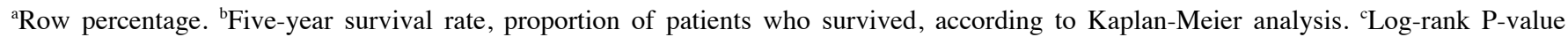
${ }^{\mathrm{d}} \mathrm{HR}, 95 \% \mathrm{CI}$ and their corresponding P-values were calculated using multivariate Cox proportional hazards model, adjusted variables included age, sex, smoking status, histological type, and pathologic stage. ${ }^{\mathrm{e} A d j u s t e d ~ v a r i a b l e s ~ i n c l u d e d ~ a g e, ~ s e x, ~ s m o k i n g ~ s t a t u s, ~ a n d ~ h i s t o l o g i c a l ~ t y p e ~}$ FOSB, FBJ murine osteosarcoma viral oncogene homolog B; NSCLC, non-small cell lung cancer; HR, hazard ratio; CI, confidence interval.

\section{Discussion}

The present study revealed that unmethylation of $F O S B$ exon 4 was detected in $18(10.2 \%)$ out of the 176 NSCLC cases and its unmethylation was related to loss of FOSB mRNA expression. The genomes of cancer cells tend to show widespread gene body hypomethylation alongside hypermethylation of gene promoter (25). Numerous studies have investigated the diagnostic and prognostic values of promoter methylation (5). However, data on the role and nature of gene body methylation in specific genes are currently limited. Thus, the present results represent the first demonstration that the gene body (exon 4) region of the FOSB gene can undergo a DNA methylation alteration in NSCLC patients. Similarly, Suzuki et al revealed that increased DNA methylation in the gene body led to the upregulation of FOSB in liver tumors of arsenic-exposed mice (24). DNA methylation in promoter sequences is well known to silence genes and is the presumed therapeutic target of methylation inhibition (26). Notably, DNA methylation is more prevalent within gene bodies than appears for promoters $(27,28)$, and gene body methylation appears to be actively involved in multiple gene regulation processes including alternative promoter usage, regulation of short and long non-coding RNAs, alternative splicing and enhancer activity (29,30), indicating a contrasting association between DNA methylation and expression across the promoter (negative association) vs. gene body (positive association).

One notable finding of the present study is the association of FOSB methylation changes with survival outcomes in a subset of patients with NSCLC, indicating that FOSB may be a new biomarker for the prognosis of NSCLC. Moreover, the present results provide evidence that FOSB may possess tumor-suppressive properties in NSCLC. These observations are in line with a study revealing that FOSB is an anti-metastatic protein in lung cancer because it negatively regulates MMP9 expression, which induces cell migration and invasion (21). Notably, the present data revealed that the unmethylation of $F O S B$ exon 4 tends to occur in patients with lymph node metastases. FOSB expression was significantly downregulated in gastric and pancreatic cancer tissues and its downregulation was associated with reduced survival $(18,19)$. FOSB overexpression also triggered cell death by regulating the expression of MMP9 in MCF breast cancer cells (31). Collectively, the present investigation indicated that FOSB exerts a tumor-suppressive function in NSCLC. The mechanism of its action requires future investigations. However, FOSB may be used as a novel biomarker or a specific therapeutic target for lung cancer.

Some of the limitations of this study include its retrospective design and the relatively small sample size, indicating that the results may be influenced by selection bias. Thus, further large-scale studies and longer follow-up periods are required to verify the clinical significance of $F O S B$ expression. Collectively, it was revealed that the decrease in DNA methylation levels of the FOSB exon 4 was associated with decreased expression of FOSB mRNA in tumor tissues and with unfavorable outcomes of patients with later stages of lung cancer. It is anticipated that accumulating knowledge on gene body methylation will provide valuable information in the future, especially to develop effective tools for DNA methylation-targeted therapy.

\section{Acknowledgements}

We would like to thank the National Biobank of Korea, KNUH for providing patient material and data.

\section{Funding}

The present study was supported by the Basic Science Research Program through the National Research Foundation of Korea (NRF) (No. NRF-2016R1D1A1B03931462). 


\section{Availability of data and materials}

The accompanying data underlying our findings are available upon request to corresponding author.

\section{Authors' contributions}

DSK and JYP drafted the manuscript, conceived and coordinated the study, and were responsible for interpretation of the data. WKL performed the statistical analyses. All authors have read and approved the final manuscript and agree to be accountable for all aspects of the research in ensuring that the accuracy or integrity of any part of the work are appropriately investigated and resolved.

\section{Ethics approval and consent to participate}

The present study was conducted with the approval of IRB, KNUH (approval no. 2014-04-210). Participants provided their written informed consent to participate in this study and for the publication of all associated data.

\section{Patient consent for publication}

Not applicable.

\section{Competing interests}

The authors declare that they have no competing interests.

\section{References}

1. Siegel RL, Miller KD and Jemal A: Cancer statistics 2018. CA Cancer J Clin 68: 7-30, 2018.

2. Spiro SG and Silvestri GA: One hundred years of lung cancer. Am J Respir Crit Care Med 172: 523-529, 2005.

3. Yano T, Haro A, Shikada Y, Maruyama R and Maehara Y: Non-Small cell lung cancer in never smokers as a representative 'non-smoking-associated lung cancer': Epidemiology and clinical features. Int J Clin Oncol 16: 287-293,2011.

4. Bird A: DNA methylation patterns and epigenetic memory. Genes Dev 16: 6-21, 2002.

5. Jankowska AM, Millward CL and Caldwell CW: The potential of DNA modifications as biomarkers and therapeutic targets in oncology. Expert Rev Mol Diagn 15: 1325-1337, 2015.

6. Lu F and Zhang HT: DNA methylation and nonsmall cell lung cancer. Anat Rec (Hoboken) 294: 1787-1795, 2011.

7. Seok Y, Lee WK, Park JY and Kim DS: TGFBI promoter methylation is associated with poor prognosis in lung adenocarcinoma patients. Mol Cells 42: 161-165, 2019.

8. Tessema M, Yingling CM, Liu Y, Tellez CS, Van Neste L, Baylin SS and Belinsky SA: Genome-Wide unmasking of epigenetically silenced genes in lung adenocarcinoma from smokers and never smokers. Carcinogenesis 35:1248-1257, 2014.

9. Shaulian E and Karin M: AP-1 as a regulator of cell life and death. Nat Cell Biol 4: E131-E136, 2002.

10. Ting $\mathrm{CH}$, Chen YC, Wu CJ and Chen JY: Targeting FOSB with a cationic antimicrobial peptide, TP4, for treatment of triple-negative breast cancer. Oncotarget 7: 40329-40347, 2016.

11. Na HH, Noh HJ, Cheong HM, Kang Y and Kim KC: SETDB1 mediated FosB expression increasing the cell proliferation rate during anticancer drug therapy. BMP Rep 49: 238-243, 2016.

12. Milde-Langosch K: The fos family of transcription factors and their role in tumorigenesis. Eur J Cancer 41: 2449-2461, 2005.
13. Barrett CS, Millena AC and Khan SA: TGF- $\beta$ effects on prostate cancer cell migration and invasion require FosB. Prostate 77: 72-81, 2017.

14. Cervantes-Madrid DL, Nagi S and Asting Gustafsson A: FosB transcription factor regulates COX-2 expression in colorectal cancer cells without affecting PGE2 expression. Oncol Lett 13: 1411-1416, 2017.

15. Shahzad MMK, Arevalo JM, Armaiz-Pena GN, Lu C, Stone RL, Moreno-Smith M, Nishimura M, Lee JW, Jennings NB, Bottsford-Miller J, et al: Stress effects on FosB- and interleukin-8 (IL8)-driven ovarian cancer growth and metastasis. J Biol Chem 285: 35462-35470, 2010.

16. Renaud SJ, Kubota K, Rumi MA and Soares MJ: The FOS transcription factor family differentially controls trophoblast migration and invasion. J Biol Chem 289: 5025-5039, 2014.

17. Rowther FB, Wei W, Dawson TP, Ashton A, Singh A, Madiesse-Timchou MP, Thomas DG, Darling JL and Warr T: Cyclic nucleotide phosphodiesterase-1C (PDE1C) drives cell proliferation, migration and invasion in glioblastoma multiform cells in vitro. Mol Carcinog 55: 268-279, 2016.

18. Tang C, Jiang Y, Shao W, Shi W, Gao X, Qin W, Jiang T, Wang F and Feng S: Abnormal expression of FOSB correlates with tumor progression and poor survival in patients with gastric cancer. Int J Oncol 49: 1489-1496, 2016.

19. Kim JH, Lee JY, Lee KT, Lee JK, Lee KH, Jang KT, Heo JS, Choi SH and Rhee JC: RGS16 and fosB underexpressed in pancreatic cancer with lymph node metastasis promote tumor progression. Tumour Biol 31: 541-548, 2010.

20. Milde-Langosch K, Kappes H, Riethdorf S, Loning T and Bamberger AM: FosB is highly expressed in normal mammary epithelia, but down-regulated in poorly differentiated breast carcinomas. Breast Cancer Res Treat 77: 265-275, 2003.

21. Wang LQ, Zhao LH and Qiao YZ: Identification of potential therapeutic targets for lung cancer by bioinformatics analysis. Mol Med Rep 13: 1975-1982, 2016.

22. Kim DS, Lee WK and Park JY: Hypermethylation of normal mucosa of esophagus-specific 1 is associated with an unfavorable prognosis in patients with non-small cell lung cancer. Oncol Lett 16: 2409-2415, 2018

23. Anier K, Malinovskaja K, Aonurm-Helm A, Zharkovsky A and Kalda A: DNA methylation regulates cocaine-induced behavioral sensitization in mice. Neuropsychopharmacol 35: 2450-2461, 2010.

24. Suzuki T, Yamashita S, Ushijima T, Takumi S, Sano T, Michikawa T and Nohara K: Genome-Wide analysis of DNA methylation changes induced by gestational arsenic exposure in liver tumors. Cancer Sci 104: 1575-1585, 2013.

25. Chen ZX and Riggs AD: DNA methylation and demethylation in mammals. J Biol Chem 286: 18347-18353, 2011.

26. Kulis M, Heath S, Bibikova M, Queiros AC, Navarro A, Clot G, Martinez-Trillos A, Castellano G, Brun-Health I, Pinyol M, et al: Epigenomic analysis detects widespread gene-body DNA hypomethylation in chronic lymphocytic leukemia. Nat Genet 44: 1236-1242, 2012

27. Yang X, Han H, De Carvalho DD, Lay FD, Jones PA and Liang G: Gene body methylation can alter gene expression and is a therapeutic target in cancer. Cancer Cell 26: 577-590, 2014.

28. Kulis M, Queiros AC, Beekman R and Martin-Subero JI: Intragenic DNA methylation in transcriptional regulation, normal differentiation and cancer. Biochim Biophys Acta 1829: 1161-1174, 2013.

29. Lee SM, Choi WY, Lee J and Kim YJ: The regulatory mechanisms of intragenic DNA methylation. Epigenomics 7: 527-531, 2015.

30. Esteller M: Dormant hypermethylated tumor suppressor genes: Questions and answers. J Pathol 205: 172-180, 2005.

31. Milde-Langosch K, Roder H, Andritzky B, Aslan B, Hemminger G, Brinkmann A, Bamberger CM, Loning T and Bamberger AM: The role of the AP-1 transcription factors c-Fos, FosB, Fra-1 and Fra-2 in the invasion process of mammary carcinomas. Breast Cancer Res Treat 86: 139-152, 2004. 